\title{
Exact Plaquette-Ordered Ground States in the Generalized Hubbard Model in Arbitrary Dimensions
}

\author{
Masaaki NAKAmURA ${ }^{1}$ and Kazuhito ITOH $^{2}$ \\ ${ }^{1}$ Department of Applied Physics, Faculty of Science, Tokyo University of Science, Tokyo 162-8601 \\ ${ }^{2}$ Department of Complex Systems Science, Graduate School of Information Science, Nagoya University, Nagoya 464-8601
}

(Received )

\begin{abstract}
We show the existance of the exact plaquette-ordered ground states of the Hubbard model including site-off-diagonal interactions in arbitrary dimensions, by decomposing the Hamiltonian as sum of products of projection operators for each spin sector. The obtatined exact ground states are interpreted as Néel ordered states on the dual lattices. We demonstrate this idea in the one-dimensional chain and higher-dimensional lattices such as the Kagomé lattice, and determine parameter regions of the exact ground states.
\end{abstract}

KEYWORDS: Hubbard model, site-off-diagonal interactions, exact ground states, bond-ordered state, plaquette-ordered state, Kagomé lattice, Pyrochlore lattice

\section{Introduction}

The Hubbard model is one of the generic models to describe interacting electrons in narrow-band systems. ${ }^{1}$ This model has played central roles to study magnetism and superconductivity. In spite of its simplicity, it is difficult to solve this model exactly except for one dimension or some special cases. On the other hand, it has been considered many extensions of the Hubbard model. The on-site repulsion of this model is due to the matrix elements of the Coulomb interaction corresponding to the on-site Wannier states, and other matrix elements are neglected. Therefore, we consider effects of these neglected terms as site-off-diagonal interactions. ${ }^{2}$ For these generalized models, exact results for ferromagnetism and superconducting states have been discussed..$^{3-9}$

Few years ago the authors discussed a different type of exact ground state in a one-dimensional system that is "bond Néel" state, ${ }^{10}$ using the decomposition of the Hamiltonian into projection operators. ${ }^{11}$ Furthermore they suggested extensions of this argument to higher dimensional systems. ${ }^{10}$ Main aim of this paper is to demonstrate the existence of the exact ground states in the generalized Hubbard model in arbitrary dimensions, especially that on the Kagomé lattice where few results have been known for Hubbard-type models. ${ }^{12,13}$

The rest of this paper is organized as follows: In sec. 2, we review the method to construct Hamiltonians with exact ground states. In sec. 3, we apply this method to the one-dimensional model discussed in ref. 10, and obtain the extended version of the phase diagram. In sec. 4, we apply the analysis to the Kagomé lattice. Finally, we give summary and discussion of the results.

\section{Method}

The strategy to construct a Hamiltonian with an exact ground state is the following way. ${ }^{11}$ First, we consider a Hamiltonian that can be decomposed as sum of products of operators for different (spin) sectors as,

$$
\mathcal{H}=\sum_{\alpha} h_{\alpha}, \quad h_{\alpha}=\sum_{\mu, \nu} \lambda_{\mu \nu} R_{\alpha \uparrow}^{(\mu)} R_{\alpha \downarrow}^{(\nu)}, \quad \lambda_{\mu \nu} \geq 0,
$$

where $\alpha$ denotes the position of one of the unit plaquettes that cover the lattice. $R_{\alpha \sigma}^{(\mu)}(\sigma=\uparrow, \downarrow)$ is an operator satisfying $\left[R_{\alpha \uparrow}^{(\mu)}, R_{\alpha \downarrow}^{(\nu)}\right]=0$. The expectation value of this operator is nonnegative $\left\langle R_{\alpha \sigma}^{(\mu)}\right\rangle \geq 0$. This condition is realized, if $R_{\alpha \sigma}^{(\mu)}$ is given by a product of an operator and its Hermitian conjugate. Then the expectation value of the Hamiltonian is also nonnegative $\langle\mathcal{H}\rangle \geq 0$.

Next, we introduce a trial wave function given by a direct product of up and down spin sectors,

$$
|\Psi(\mathcal{A}, \mathcal{B})\rangle=\left|\Phi_{\uparrow}(\mathcal{A})\right\rangle \otimes\left|\Phi_{\downarrow}(\mathcal{B})\right\rangle
$$

where $\mathcal{A}$ and $\mathcal{B}$ denote two groups of plaquettes that cover the lattice satisfying $\mathcal{A} \cup \mathcal{B}=\{$ all lattice sites $\}$. We require that the projection operators have the following conditions,

$$
R_{\alpha \uparrow}^{(\mu)}\left|\Phi_{\uparrow}(\mathcal{A})\right\rangle=R_{\beta \downarrow}^{(\mu)}\left|\Phi_{\downarrow}(\mathcal{B})\right\rangle=0,
$$

where $\alpha \in \mathcal{A}$ and $\beta \in \mathcal{B}$. Note that

$$
R_{\beta \uparrow}^{(\mu)}\left|\Phi_{\uparrow}(\mathcal{A})\right\rangle \neq 0, \quad R_{\alpha \downarrow}^{(\mu)}\left|\Phi_{\downarrow}(\mathcal{B})\right\rangle \neq 0 .
$$

Therefore, the eigenvalue of the Hamiltonian for $|\Psi(\mathcal{A}, \mathcal{B})\rangle$ is always zero. Then, the lower bound and the upper bound of the energy coincide, so that $|\Psi(\mathcal{A}, \mathcal{B})\rangle$ turns out to be one of the exact ground state of this system.

The above argument is satisfied in corner sharing lattices with the bipartite structure in terms of their dual lattices. For example, in two dimensions, the Kagomé (Checkerboard) lattice can be covered by two plaquettes for different sectors alternatively, as illustrated in fig. 1 . These states can be regarded as the Néel ordering on the honeycomb (square) lattice. In three dimensions, the Pyrochlore lattice and the Garnet lattice ${ }^{14}$ satisfy these conditions.

\section{One-dimensional chain}

First, we consider the one-dimensional generalized Hubbard model at half-filling and zero-magnetic field, 


$$
\propto-\frac{\downarrow}{i} \underset{j}{\infty} \underset{j}{-} \underset{\uparrow}{\longrightarrow} \quad \text { Chain }
$$
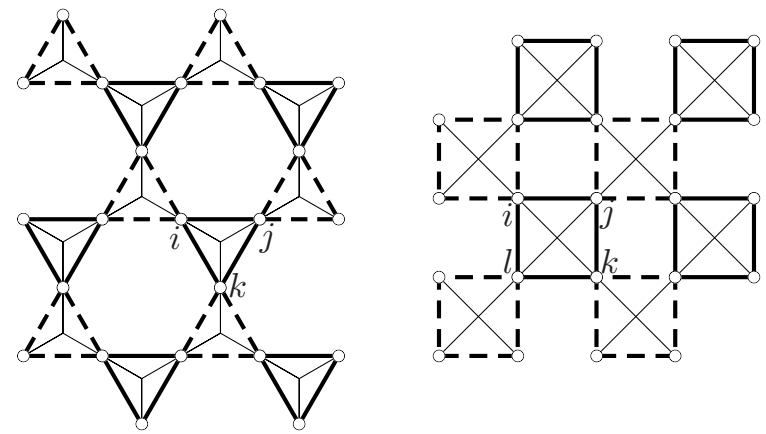

Kagomé

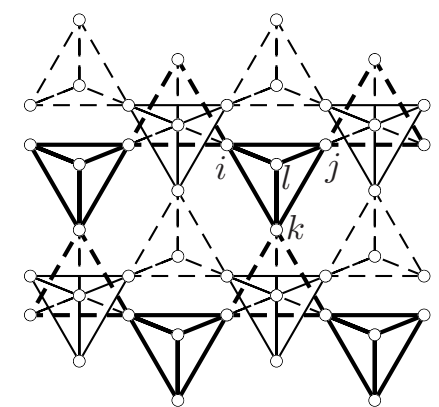

Pyrochlore

Fig. 1. Examples of lattice structure where generalized Hubbard models with exact plaquette-ordered ground states can be constructed: the one-dimensional chain, the Kagomé, the Checkerboard and the Pyrochlore lattices. The solid and the dashed plaquettes denote those belong to the groups $\mathcal{A}$ and $\mathcal{B}$, respectively.

given by $\mathcal{H}=\sum_{i \sigma} h_{i, i+1, \sigma}$ with the local bond Hamiltonian,

$$
\begin{aligned}
h_{i j \sigma}= & -t T_{i j \sigma}+\frac{U}{2 z}\left(n_{i \sigma} n_{i \bar{\sigma}}+n_{j \sigma} n_{j \bar{\sigma}}\right) \\
& +V_{\|} n_{i \sigma} n_{j \sigma}+V_{\perp} n_{i \sigma} n_{j \bar{\sigma}} \\
& +X T_{i j \sigma}\left(n_{i \bar{\sigma}}+n_{j \bar{\sigma}}\right)+\frac{W}{2} \sum_{\sigma^{\prime}} T_{i j \sigma} T_{i j \sigma^{\prime}}
\end{aligned}
$$

where $\bar{\sigma}$ is the opposite spin of $\sigma$, and periodic boundary conditions are assumed. The number of the nearest sites is $z=2$. We have defined the hopping and the density operators as $T_{i j \sigma} \equiv c_{i \sigma}^{\dagger} c_{j \sigma}+$ H.c., $n_{i \sigma} \equiv c_{i \sigma}^{\dagger} c_{i \sigma}$. Note that the bond-bond interaction $(W)$ term can be rewritten as

$$
-2 W\left(\boldsymbol{S}_{i} \cdot \boldsymbol{S}_{j}+\boldsymbol{\eta}_{i} \cdot \boldsymbol{\eta}_{j}-\frac{1}{4}\right),
$$

where $\boldsymbol{S}_{i}$ and $\boldsymbol{\eta}_{i}$ are the spin and the pseudo spin operators, respectively. The components of the pseudo spin operator are defined by

$\eta_{i}^{+} \equiv(-1)^{i} c_{i \uparrow}^{\dagger} c_{i \downarrow}^{\dagger}, \quad \eta_{i}^{-} \equiv(-1)^{i} c_{i \downarrow} c_{i \uparrow}, \quad \eta_{i}^{z} \equiv \frac{1}{2}\left(n_{i \uparrow}+n_{i \downarrow}-1\right)$.

Now, we introduce bond operators corresponding to the bonding and the anti-bonding modes,

$$
A_{i j \sigma}^{\dagger}=\frac{1}{\sqrt{2}}\left(c_{i \sigma}^{\dagger}+c_{j \sigma}^{\dagger}\right), \quad B_{i j \sigma}^{\dagger}=\frac{1}{\sqrt{2}}\left(c_{i \sigma}^{\dagger}-c_{j \sigma}^{\dagger}\right) .
$$

The two electron states are described as $B_{i j \sigma}^{\dagger} A_{i j \sigma}^{\dagger}=$ $c_{i \sigma}^{\dagger} c_{j \sigma}^{\dagger}$. These operators on the same bond satisfy the anticommutation relations:

$$
\left\{A_{i j \sigma}, A_{i j \sigma^{\prime}}^{\dagger}\right\}=\left\{B_{i j \sigma}, B_{i j \sigma^{\prime}}^{\dagger}\right\}=\delta_{\sigma \sigma^{\prime}}, \quad \text { otherwise }=0 .
$$

The density operators for the bond operators are given as

$$
\begin{aligned}
& n_{A \sigma} \equiv A_{i j \sigma}^{\dagger} A_{i j \sigma}=\frac{1}{2}\left(n_{i \sigma}+n_{j \sigma}+T_{i j \sigma}\right), \\
& n_{B \sigma} \equiv B_{i j \sigma}^{\dagger} B_{i j \sigma}=\frac{1}{2}\left(n_{i \sigma}+n_{j \sigma}-T_{i j \sigma}\right) .
\end{aligned}
$$

Since we restrict our attention only on the neighboring two sites $i, j$, we drop these indices from the operators defined above.

As a trial state, we consider the following wave function,

$$
\left|\Psi_{\sigma}\right\rangle \equiv A_{12 \sigma}^{\dagger} A_{23 \bar{\sigma}}^{\dagger} \cdots A_{L-1, L \sigma}^{\dagger} A_{L, 1 \bar{\sigma}}^{\dagger}|0\rangle,
$$

where $|0\rangle$ denotes a vacuum and $L$ is the number of sites. This state is regarded as a Néel ordering of the bondlocated spins, so that we call this bond Néel (BN) state. There is two-fold degeneracy given by $\left|\Psi_{\uparrow}\right\rangle$ and $\left|\Psi_{\downarrow}\right\rangle$. In order to construct a model with the exact ground state, the local Hamiltonian $h_{i j}=\sum_{\sigma} h_{i j \sigma}$ should be decomposed by the projection operators $1-n_{A \sigma}$ and $n_{B \sigma}$ (see table I) in the following form,

$$
\begin{aligned}
h_{i j}- & \varepsilon_{0}=\lambda_{\bar{A} \bar{A}}\left(1-n_{A \uparrow}\right)\left(1-n_{A \downarrow}\right)+\lambda_{B B} n_{B \uparrow} n_{B \downarrow} \\
& +\lambda_{\bar{A} B}\left\{\left(1-n_{A \uparrow}\right) n_{B \downarrow}+n_{B \uparrow}\left(1-n_{A \downarrow}\right)\right\}, \quad(12
\end{aligned}
$$

where $\varepsilon_{0}$ is the ground-state energy par bond. According to the argument given in sec. 2, for the BN ground state, the parameters should be chosen as

$$
\lambda_{\bar{A} \bar{A}}, \quad \lambda_{\bar{A} B}, \quad \lambda_{B B} \geq 0 .
$$

Comparing eqs. (5) and (12), the relations among the parameters are obtained as

$$
V_{\perp}=\frac{U}{2}, \quad V_{\|}=W, \quad X=t-W .
$$

The parameters in eq. (12) are identified as follows,

$$
\begin{aligned}
& \lambda_{\bar{A} \bar{A}}=\frac{U}{2}-W+2 t, \\
& \lambda_{\bar{A} B}=-\frac{U}{2}+W, \\
& \lambda_{B B}=\frac{U}{2}+3 W-2 t .
\end{aligned}
$$

From eqs. (13), (15), (16) and (17), we obtain the parameter space of the BN ground state as shown in fig. 2 . Note that the BN state appears only for $t>0$ region.

\begin{tabular}{|c|cccc|}
\hline & $|0\rangle$ & $A_{\sigma}^{\dagger}|0\rangle$ & $B_{\sigma}^{\dagger}|0\rangle$ & $B_{\sigma}^{\dagger} A_{\sigma}^{\dagger}|0\rangle$ \\
\hline $1-n_{A \sigma}$ & 1 & 0 & 1 & 0 \\
$n_{B \sigma}$ & 0 & 0 & 1 & 1 \\
\hline
\end{tabular}

Table I. Projection operators to construct the Hamiltonian on the one-dimensional chain with the bond Néel (BN) ground state. We can also discuss the parameter region of the ferromagnetic (FM) and phase-separated (PS) states. 


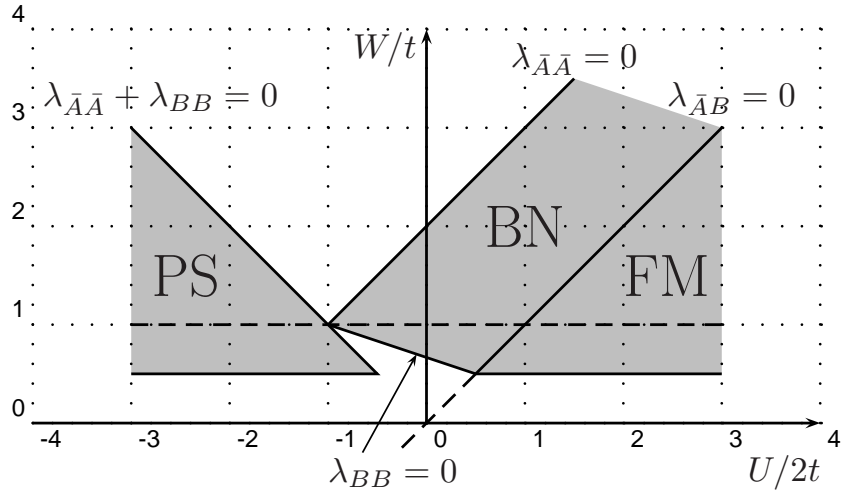

Fig. 2. Phase diagrams of the generalized Hubbard chain (5) in the $U / 2 t-W / t$ parameter space with $t>0 .{ }^{10,15}$ The other parameters are set as $X=t-W, V_{\|}=W$ and $V_{\perp}=U / 2$. The shaded regions labelled by BN, FM and PS denote bond-Néel, ferromagnetic and phase-separated states, respectively.

The property of the BN state can be investigated based on the matrix-product method. According to ref. 10, both charge-charge and spin-spin correlation functions vanish except for those of the nearest sites which indicates the existence of the charge and the spin gaps. On the other hand, the bond-located spin correlation exhibits a long range order. We can also calculate elementally excitation spectrum using the matrix-product method as a variational approach. ${ }^{15}$

In the present one-dimensional model at half-filling, we can discuss not only the BN state but also the ferromagnetic (FM) and the phase-separated (PS) states. As shown in table I, the last term of eq. (12) stabilizes the fully polarized FM state for $\lambda_{\bar{A} B}<0$. Similarly, the PS state where the system is separated into a domain of doubly occupied sites and a vacuum, is stabilized when $\lambda_{\bar{A} \bar{A}}+\lambda_{B B}<0$, neglecting the surface energy. As shown in fig. 2, the FM and the PS states appear in the $U / 2 t$ $W / t$ parameter space symmetrically in the positive- and in the negative- $U$ regions, respectively. This is consistent with the fact that the $W$ term is the ferromagnetic exchange interactions of the spins and the pseudo spins (6), and the PS state is regarded as the FM state of the pseudo-spin space. The condition $W / t \geq 1 / 2$ for the FM and the PS phases is not clearly obtained in the present argument. This will be discussed in Sec. 5 .

The phase boundary of the BN and the FM states $\lambda_{\bar{A} B}=0$ corresponds to the $\mathrm{SU}(2)$ symmetry in the spins $V_{\|}=V_{\perp}$, so that the ground state is highly degenerate. The system undergoes a first-order phase transition at this level-crossing point. When $W / t=1(X=0)$, there is the particle-hole symmetry. At $(U / 2 t, W / t)=(-1,1)$, the system has the $\mathrm{SU}(2)$ symmetry in the pseudo-spin space, so that the $\mathrm{BN}$, the PS and the $\eta$-paring states are degenerate. The other lines which separate shaded and non-shaded regions in fig. 2 do not necessarily mean phase boundaries.

\section{Kagomé lattice}

We consider the generalized Hubbard model including three site terms on the Kagomé lattice at 1/3-filling with zero-magnetic field, $\mathcal{H}=\sum_{\langle i j k\rangle \sigma} h_{i j k \sigma}$, where the sum- mation $\langle i j k\rangle$ is taken in each unit trimer as shown in fig. 1,

$$
\begin{aligned}
h_{i j k \sigma} & =h_{i j \sigma}+h_{j k \sigma}+h_{k i \sigma} \\
& +W^{\prime}\left(T_{i j \sigma} T_{j k \bar{\sigma}}+T_{j k \sigma} T_{k i \bar{\sigma}}+T_{k i \sigma} T_{i j \bar{\sigma}}\right) \\
& +X^{\prime}\left(T_{i j \sigma} n_{k \bar{\sigma}}+T_{j k \sigma} n_{i \bar{\sigma}}+T_{k i \sigma} n_{j \bar{\sigma}}\right),
\end{aligned}
$$

where $h_{i j \sigma}$ is the local bond Hamiltonian defined in eq. (5) for $z=4$. The three site interactions $\left(W^{\prime}, X^{\prime}\right)$ have the similar structure to those of the $t-J$ model.

We define the following one-electron plaquette operators corresponding to a zero momentum mode, left and right chirality modes,

$$
\begin{aligned}
A_{i j k \sigma}^{\dagger} & =\frac{1}{\sqrt{3}}\left(c_{i \sigma}^{\dagger}+c_{j \sigma}^{\dagger}+c_{k \sigma}^{\dagger}\right), \\
B_{i j k \sigma}^{\dagger} & =\frac{1}{\sqrt{3}}\left(c_{i \sigma}^{\dagger}+\omega c_{j \sigma}^{\dagger}+\omega^{2} c_{k \sigma}^{\dagger}\right), \\
C_{i j k \sigma}^{\dagger} & =\frac{1}{\sqrt{3}}\left(c_{i \sigma}^{\dagger}+\omega^{2} c_{j \sigma}^{\dagger}+\omega c_{k \sigma}^{\dagger}\right),
\end{aligned}
$$

where $\omega=\mathrm{e}^{\mathrm{i} 2 \pi / 3}$. These operators on the same trimer satisfy the anticommutation relations:

$\left\{A_{i j k \sigma}, A_{i j k \sigma^{\prime}}^{\dagger}\right\}=\left\{B_{i j k \sigma}, B_{i j k \sigma^{\prime}}^{\dagger}\right\}=\left\{C_{i j k \sigma}, C_{i j k \sigma^{\prime}}^{\dagger}\right\}=\delta_{\sigma \sigma^{\prime}}$,

and otherwise $=0$. Note that $A_{i j k \sigma}^{\dagger}|0\rangle, B_{i j k \sigma}^{\dagger}|0\rangle$ and $C_{i j k \sigma}^{\dagger}|0\rangle$ are chosen as eigenstates of density, hopping and current operators:

$$
\begin{aligned}
N_{i j k \sigma} & \equiv n_{i \sigma}+n_{j \sigma}+n_{k \sigma}, \\
T_{i j k \sigma} & \equiv T_{i j \sigma}+T_{j k \sigma}+T_{k i \sigma}, \\
J_{i j k \sigma} & \equiv J_{i j \sigma}+J_{j k \sigma}+J_{k i \sigma}
\end{aligned}
$$

where $J_{i j \sigma} \equiv \mathrm{i}\left(c_{i \sigma}^{\dagger} c_{j \sigma}-\right.$ H.c. $)$. The density operators for the plaquette operators are

$$
\begin{aligned}
n_{A \sigma} & =\frac{1}{3}\left(N_{i j k \sigma}+T_{i j k \sigma}\right), \\
n_{B \sigma} & =\frac{1}{6}\left(2 N_{i j k \sigma}-T_{i j k \sigma}-\sqrt{3} J_{i j k \sigma}\right), \\
n_{C \sigma} & =\frac{1}{6}\left(2 N_{i j k \sigma}-T_{i j k \sigma}+\sqrt{3} J_{i j k \sigma}\right) .
\end{aligned}
$$

Now we consider the Néel ordered state given by $A_{i j k \sigma}^{\dagger}$ on the honeycomb lattice as shown in fig. 1 . Then the local Hamiltonian $h_{i j k}=\sum_{\sigma} h_{i j k \sigma}$ corresponding to this state consists of projection operators $1-n_{A \sigma}, n_{B \sigma}$ and $n_{C \sigma}$. In order to eliminate the current term from the Hamiltonian, we construct $h_{i j k}$ in the following way,

$$
\begin{aligned}
h_{i j k} & -\varepsilon_{0}=\lambda_{\bar{A} \bar{A}}\left(1-n_{A \uparrow}\right)\left(1-n_{A \downarrow}\right) \\
& +\lambda_{\bar{A} B}\left\{\left(1-n_{A \uparrow}\right)\left(n_{B \downarrow}+n_{C \downarrow}\right)+\left(n_{B \uparrow}+n_{C \uparrow}\right)\left(1-n_{A \downarrow}\right)\right\} \\
& +\lambda_{B B}\left(n_{B \uparrow}+n_{C \uparrow}\right)\left(n_{B \downarrow}+n_{C \downarrow}\right),
\end{aligned}
$$

where the parameters should satisfy the condition of eq. (13). Comparing eqs. (18) and (28), the relations among the parameters are obtained as

$$
V_{\perp}=\frac{U}{2}, \quad V_{\|}=W=W^{\prime}, \quad X=X^{\prime}=t-2 W,
$$




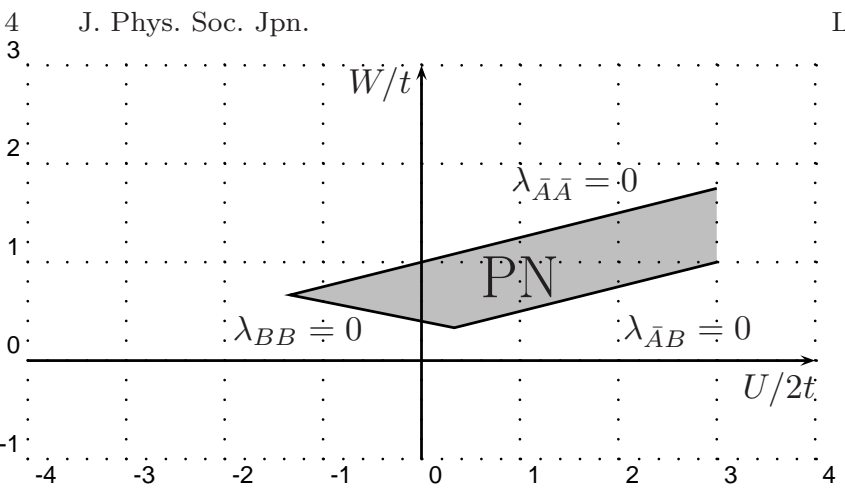

Fig. 3. Phase diagram of the generalized Hubbard model on the Kagomé lattice (18), in the $U / 2 t-W / t$ parameter space with $V_{\|}=$ $W=W^{\prime}, V_{\perp}=U / 2, X=X^{\prime}=t-2 W$ and $t>0$. The shaded region labelled by $\mathrm{PN}$ denotes the plaquette Néel state.

and parameters in eq. (28) are identified as

$$
\begin{aligned}
& \lambda_{\bar{A} \bar{A}}=\frac{U}{2}-4 W+4 t \\
& \lambda_{\bar{A} B}=-\frac{U}{2}+4 W-t, \\
& \lambda_{B B}=\frac{U}{2}+5 W-2 t .
\end{aligned}
$$

Thus the parameter region of the exact ground state with the plaquette long-range order is obtained as shown in fig. 3 .

We can extend the parameter space of the same exact ground state, introducing more parameters in eq. (28), such as $\lambda_{B B}\left(n_{B \uparrow}+n_{C \uparrow}\right)\left(n_{B \downarrow}+n_{C \downarrow}\right) \rightarrow \lambda_{B B}\left(n_{B \uparrow} n_{B \downarrow}+\right.$ $\left.n_{C \uparrow} n_{C \downarrow}\right)+\lambda_{B B}^{\prime}\left(n_{B \uparrow} n_{C \downarrow}+n_{C \uparrow} n_{B \downarrow}\right)$. Moreover, it is also possible to construct models for chiral states given by $B_{i j k \sigma}^{\dagger}$ and $C_{i j k \sigma}^{\dagger}$, for the ferromagnetic state at halffilling, and for two-electron plaquette states at $2 / 3$-filling such as

$$
C_{i j k \sigma}^{\dagger} B_{i j k \sigma}^{\dagger}=\frac{\mathrm{i}}{\sqrt{3}}\left(c_{i \sigma}^{\dagger} c_{j \sigma}^{\dagger}+c_{j \sigma}^{\dagger} c_{k \sigma}^{\dagger}+c_{k \sigma}^{\dagger} c_{i \sigma}^{\dagger}\right) .
$$

\section{Summary and discussion}

We have discussed a method to construct generalized Hubbard Hamiltonians with exact plaquette-ordered ground states in arbitrary dimensions. The corresponding lattices have bipartite structure in terms of corner sharing unit plaquettes. We have applied this method to the one-dimensional chain and the Kagomé lattice, and obtained parameter regions of the exact ground states.

In one dimension, the $\mathrm{BN}$ state corresponds to the staggered dimer states in the spin-1/2 two-leg ladder model with four spin exchanges. ${ }^{16}$ Comparing with the way to decompose the Hamiltonian to the projection operators in ref. 16, the present argument is quite simple. This is because the generalized Hubbard models we have

\begin{tabular}{|c|cccc|}
\hline & $|0\rangle$ & $A_{\sigma}^{\dagger}|0\rangle$ & $B_{\sigma}^{\dagger}|0\rangle$ & $B_{\sigma}^{\dagger} A_{\sigma}^{\dagger}|0\rangle$ \\
\hline$\left(1-n_{A \sigma}\right)\left(1-n_{B \sigma}\right)$ & 1 & 0 & 0 & 0 \\
$\left(1-n_{A \sigma}\right) n_{B \sigma}$ & 0 & 0 & 1 & 0 \\
$n_{A \sigma} n_{B \sigma}$ & 0 & 0 & 0 & 1 \\
\hline
\end{tabular}

Table II. A different set of projection operators for the onedimensional chain with the $\mathrm{BN}$ ground state. considered do not include hopping terms between different spin sectors.

Application of this method to other lattices can be done straightforwardly. The bases of plaquette operators should be chosen reflecting symmetry of lattices. For example, the unit plaquettes of the Checkerboard and the Pyrochlore lattices consist of four sites, but the bases of the plaquette operators are chosen in different way. Similarly, we can also construct Heisenberg-type models with plaquette-ordered ground states in these lattice systems. These ground states have two-fold degeneracy, but the uniqueness has not been proven yet.

In this paper, we have decomposed particular Hamiltonians into the projection operators. However, we need more general treatment to decompose the arbitrary given Hamiltonians. In the present case, one projection operator gives positive value for more than two states as summarized in table. I. To remove this overlap, we should redefine the projection operators. For example, in onedimensional case, we should choose three operators to treat arbitrary bond Hamiltonians as shown in table II. Then the number of free parameters becomes six. On the other hand, in sec. 3, we have introduced three parameters. Among these three extra parameters, the hidden conditions for the ground states $t>0$ and $W / t \geq 1 / 2$ are included. Moreover, when we add the three site term $T_{i j \sigma} n_{i \bar{\sigma}} n_{j \bar{\sigma}}$ to the model, these hidden conditions are essential to determine the regions of the $\mathrm{BN}$ ground state. The detail of this argument will be published elsewhere. ${ }^{15}$

\section{Acknowledgments}

M. N. thanks Y. Motome and K. Penc for stimulating discussions. M. N. is partly supported by the Grant-inAid for scientific research of the Ministry of Education, Science, Sports and Culture of Japan.

1) J. Hubbard: Proc. R. Soc. London A 276 (1963) 238; J. Kanamori: Prog. Theor. Phys. 30 (1963) 275; M. C. Gutzwiller: Phys. Rev. Lett. 10 (1963) 159.

2) D. K. Campbell, J. T. Gammel and E. W. Loh, Jr.: Phys. Rev. B 38 (1988) 12043; 42 (1990) 475.

3) R. Strack and D. Vollhardt: Phys. Rev. Lett. 70 (1993) 2637.

4) R. Strack and D. Vollhardt: Phys. Rev. Lett. 72 (1994) 3425.

5) L. Arrachea and A.A. Aligia: Phys. Rev. Lett. 73 (1994) 2240.

6) J. de Boer, V.E. Korepin and A. Schadschneider: Phys. Rev. Lett. 74 (1995) 789.

7) J. de Boer and A. Schadschneider: Phys. Rev. Lett. 75 (1995) 4298.

8) A. Montorsi and D. K. Campbell: Phys. Rev. B 53 (1996) 5153

9) M. Kollar, R. Strack and D. Vollhardt: Phys. Rev. B 53 (1996) 9225.

10) K. Itoh, M. Nakamura and N. Muramoto: J. Phys. Soc. Jpn. 70 (2001) 1202.

11) K. Itoh: J. Phys. Soc. Jpn. 68 (1999) 322.

12) A. Tanaka and H. Ueda: Phys. Rev. Lett. 90 (2003) 067204.

13) Y. Imai, N. Kawakami and H. Tsunetsugu: Phys. Rev. B 68 (2003) 195103.

14) for example, O. A. Petrenko and D. McK. Paul: Phys. Rev. B 63 (2001) 024409.

15) M. Nakamura, T. Okano and K. Itoh: unpublished.

16) A. K. Kolezhuk and H.-J. Mikeska: Phys. Rev. Lett. 80 (1998) 2709; Int. J. Mod. Phys. B 12 (1998) 2325. 